\title{
Simultaneous Measurements of Strain and Temperature Using Long-period Fiber Grating Written on Twisted High-birefringence Photonic Crystal Fiber
}

\author{
Do Kyung Kim, ${ }^{1}$ Jihoon Kim, ${ }^{2}$ Seul-Lee Lee, ${ }^{1}$ Sungwook Choi, ${ }^{1}$ \\ Min Seok Kim, ${ }^{1}$ Jinsil Han, ${ }^{2}$ and Yong Wook Lee ${ }^{1,2^{*}}$ \\ ${ }^{1}$ Interdisciplinary Program of Biomedical Mechanical and Electrical Engineering, Pukyong National University, \\ 45 Yongso-ro, Nam-gu, Busan 48513, Korea \\ ${ }^{2}$ School of Electrical Engineering, Pukyong National University, \\ 45 Yongso-ro, Nam-gu, Busan 48513, Korea
}

(Received February 14, 2020; accepted April 20, 2020)

Keywords: long-period fiber grating, high-birefringence photonic crystal fiber, twisted fiber, strain, temperature, simultaneous measurement

Here, we propose a novel fiber-optic sensor capable of simultaneous measurements of strain and temperature by using a long-period fiber grating (LPFG) inscribed on a twisted highbirefringence photonic crystal fiber (HBPCF) as a sensor head. The sensor head was fabricated by scanning $\mathrm{CO}_{2}$ laser pulses to one side of the twisted HBPCF using a line-by-line technique. The LPFG written on the twisted HBPCF (referred to as a THBPC-LPFG) exhibits two different resonance bands, obtained at two orthogonal input polarization states, with multiple attenuation dips within each band. Two attenuation dips, each of which was selected in each resonance band, were utilized as sensor indicators. For these two indicator dips designated as Dips 1 and 2 , their strain and temperature responses were examined in a strain range of 0-1953 $\mu \varepsilon$ with a step of $217 \mu \varepsilon$ and in a temperature range of $35-95{ }^{\circ} \mathrm{C}$ with a step of $10{ }^{\circ} \mathrm{C}$. At a fixed room temperature $\left(25^{\circ} \mathrm{C}\right)$, that is, without any temperature changes, the strain sensitivities of Dips 1 and 2 were measured to be approximately -0.99 and $-1.29 \mathrm{pm} / \mu \varepsilon$, respectively. Similarly, the temperature sensitivities of Dips 1 and 2 were measured and found to be $\sim 7.14$ and $\sim 10.07$ $\mathrm{pm} /{ }^{\circ} \mathrm{C}$ without the applied strain, respectively. Owing to their linear and independent responses to strain and temperature, strain and temperature changes applied to the THBPC-LPFG can be simultaneously estimated from the measured wavelength shifts of the two indicator dips (i.e., Dips 1 and 2) using their predetermined strain and temperature sensitivities. From the repetitive simultaneous measurements of the applied strain and temperature, the rms deviations of the measured strain and temperature were evaluated as $20.2 \mu \varepsilon$ and $1.78{ }^{\circ} \mathrm{C}$, respectively. The experimental results prove that the THBPC-LPFG can be employed as a cost-effective sensor head for the simultaneous measurements of strain and temperature.

*Corresponding author: e-mail: yongwook@pknu.ac.kr https://doi.org/10.18494/SAM.2020.2829 


\section{Introduction}

Over the past decade, fiber-optic sensors have demonstrated the potential for applications in a wide range of fields, such as structural health and environmental monitoring and biomedical instrumentation, owing to their merits of rapid response, compactness, durability, and immunity to electromagnetic interference, compared with conventional electronic sensors. Optical fiber gratings, which are intrinsic fiber devices that allow the modulation of the properties of light propagating within the fiber, are most commonly employed as the sensor heads of fiber-optic sensors. According to the grating period, fiber gratings are classified into short-period fiber gratings, also called fiber Bragg gratings (FBGs), with periodicities on the order of the optical wavelength, and long-period fiber gratings (LPFGs) with periodicities of 100-1000 nanometers. In particular, LPFGs enable the optical power coupling between the fundamental core mode and the copropagating cladding modes at specific resonance wavelengths. This mode coupling results in the transmission spectrum of an LPFG, which has a series of rejection bands centered at discrete resonance wavelengths. Each rejection band is formed by the coupling of the fundamental core mode into one of the dissimilar cladding modes. In comparison with those of FBGs, the resonance dips of LPFGs are much more sensitive to external perturbations, such as curvature, ${ }^{(1-3)}$ surrounding refractive index, ${ }^{(4,5)}$ humidity, ${ }^{(6,7)}$ strain, ${ }^{(8-10)}$ and temperature. ${ }^{(11-13)}$ However, one of the main challenges of LPFGs is their cross-sensitivity between strain and temperature. To eliminate this cross sensitivity, numerous approaches have been suggested to realize separate measurements of strain and temperature. ${ }^{(14-16)}$ Among these works, an LPFG written on a conventional high-birefringence fiber (HBF), designated as an HB-LPFG, provided an efficient and affordable solution to the simultaneous measurements of strain and temperature. ${ }^{(16)}$ It was possible to obtain two resonance dips with dissimilar cladding-mode orders, obtained at two orthogonal input polarization states (PSs) aligned along the principal axes of the HB-LPFG, i.e., slow and fast axes, within several tens of nanometers. ${ }^{(16)}$ The two resonance dips with different cladding-mode orders have different strain and temperature sensitivities as well. The simultaneous measurements of strain and temperature could be accomplished by harnessing their independent strain and temperature responses. The birefringence of a conventional HBF is introduced by two stress-applying parts placed on either side of the core, which have a thermal expansion coefficient different from that of silica. The elasto-optically induced HBF birefringence can easily be varied by bending, leading to the coupling between orthogonal polarization modes. This bend-induced mode coupling deteriorates the stability and accuracy of the sensor when an HBF is employed as a sensing fiber on which an LPFG is written. To resolve this mode-coupling problem, a high-birefringence photonic crystal fiber (HBPCF) composed only of pure silica can be utilized as a sensing fiber. If an LPFG written on an HBPCF is employed as a sensor head, the mechanical stability of the sensor can be improved because the elliptical core of the HBPCF creates a stronger birefringence (close to $10^{-3}$ ) than that of the conventional HBF (e.g., $\sim 5.05 \times 10^{-4}$ ), ${ }^{(16)}$ which suppresses the mode coupling between orthogonal PSs, induced by macro- or microbending. Moreover, the measurement accuracy, another important performance parameter, can be enhanced by sharpening the resonance dip of an LPFG, whose sharpness normally increases with decreasing bandwidth. When an LPFG 
is fabricated on a twisted fiber, the spectral splitting of its individual resonance dips occurs, ${ }^{(17)}$ which leads to multiple attenuation dips with smaller bandwidths in each resonance dip. These attenuation dips with smaller bandwidths have more pointed dips beneficial to the accurate measurement of their wavelength shifts.

Here, we propose a fiber-optic sensor capable of simultaneously measuring strain and temperature using an LPFG inscribed on a twisted HBPCF, referred to as a THBPC-LPFG, by $\mathrm{CO}_{2}$ laser pulses as a sensor head. As far as we know, this is the first study on the application of the THBPC-LPFG to the simultaneous measurements of strain and temperature. The transmission spectrum of the fabricated THBPC-LPFG has two different rejection bands according to the input PS, each of which has multiple attenuation dips within its band. Two pointed attenuation dips, one dip at each rejection band, obtained at two orthogonal input PSs, were chosen as sensor indicators designated as Dips 1 and 2. The axial strain and temperature responses of these indicators of the fabricated THBPC-LPFG were investigated by applying independent strain and temperature variations (denoted by $\Delta S$ and $\Delta T$ ) to the sensor head, respectively. When the axial strain was applied (i.e., $\Delta S>0$ ) to the THBPC-LPFG at a fixed room temperature $(\Delta T=0)$, Dips 1 and 2 , whose wavelengths were indicated by $\lambda_{1}$ and $\lambda_{2}$, respectively, showed blue shifts of different amounts. On the other hand, with increasing ambient temperature $(\Delta T>0)$ without the applied strain $(\Delta S=0), \lambda_{1}$ and $\lambda_{2}$ exhibited red shifts of different amounts. On the basis of the linear responses of $\lambda_{1}(\Delta S, \Delta T)$ and $\lambda_{2}(\Delta S, \Delta T)$ with respect to strain and temperature and the independence of $\lambda_{1}(\Delta S, \Delta T)$ and $\lambda_{2}(\Delta S, \Delta T)$ responses, strain and temperature variations applied to the THBPC-LPFG (i.e., $\Delta S$ and $\Delta T$ ) can be simultaneously derived from the measured wavelength shifts of Dips 1 and 2 utilizing the predetermined strain and temperature sensitivities of $\lambda_{1}$ and $\lambda_{2}$ (i.e., $\Delta \lambda_{1} / \Delta S, \Delta \lambda_{2} / \Delta S, \Delta \lambda_{1} / \Delta T$, and $\Delta \lambda_{2} / \Delta T$ ). In Sect. 2, the fabrication process and spectral characteristics of the THBPCLPFG will be described. Then, experimental results on the strain and temperature responses of the THBPC-LPFG and some discussion on them will be provided in Sect. 3. Finally, a brief summary and conclusion will be given in Sect. 4.

\section{Fabrication and Spectral Characteristics of THBPC-LPFG and Principle of Simultaneous Measurement}

The mode-field diameters of an HBPCF (Crystal Fibre) on which an LPFG is written are 4.3 and $6.8 \mu \mathrm{m}$ along its slow and fast axes, and its birefringence and attenuation are $\sim 8.18 \times 10^{-4}$ and less than $2 \mathrm{~dB} / \mathrm{km}$ at $1550 \mathrm{~nm}$, respectively. An HBPCF is well known for a reduced bendinduced coupling between orthogonal polarization modes and a 30 times lower temperature sensitivity than a conventional HBF. Figure 1 shows a schematic diagram of the fabrication setup of a THBPC-LPFG based on a $10.6 \mu \mathrm{m} \mathrm{CO}_{2}$ laser (Synrad Inc.). The THBPC-LPFG was fabricated by forming an LPFG on an unjacketed HBPCF, twisted at a rate of $R_{T}=2 \pi N / L,{ }^{(17)}$ with $\mathrm{CO}_{2}$ laser pulses. Here, $N$ and $L$ are the number of twist turns and the length of the twisted fiber, respectively. The diameter of the output beam of the $\mathrm{CO}_{2}$ laser is increased by the beam expander, and the expanded beam is focused by an $f-\theta$ lens in a computer-controlled 2D optical scanner. Then, the focused beam with a spot size of $\sim 70 \mu \mathrm{m}$ is exposed to the HBPCF. The 


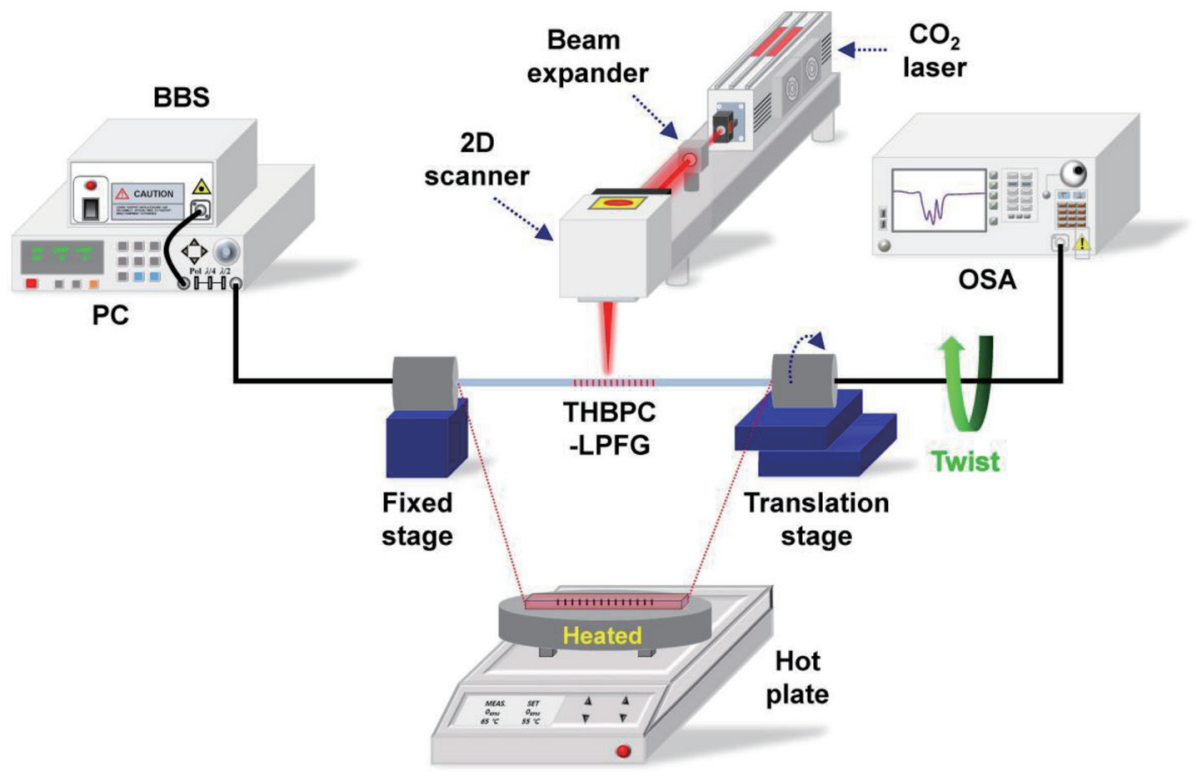

Fig. 1. (Color online) Schematic diagram of inscription setup of the THBPC-LPFG and experimental setup used to investigate its strain and temperature responses.

average power of the focused $\mathrm{CO}_{2}$ laser beam was $\sim 2.23 \mathrm{~W}$. The grating pitch, grating length, number of laser scanning cycles, and twist rate $\left(R_{T}\right)$ of the fabricated THBPC-LPFG were $\sim 400 \mu \mathrm{m}, 12.0 \mathrm{~mm}$ (30 grating periods), 6 cycles, and $\sim 31.42 \mathrm{~m}^{-1}$, respectively. During the laser irradiation of the HBPCF segment, one end of the segment was fixed, and longitudinal tension was applied to the other end with a weight of $15 \mathrm{~g}$ to ensure the exact grating period of the fabricated THBPC-LPFG. Moreover, during the whole process of the grating fabrication, the transmission spectrum of the fabricated grating was monitored by using a broadband light source (BBS, Fiberlabs FL7701), a polarization controller (PC, Agilent 8169A), and an optical spectrum analyzer (OSA, Yokogawa AQ6370C), as shown in Fig. 1.

The phase-matching condition of a conventional LPFG can be described as

$$
\lambda_{\text {res }}=\left(n_{c o, e f f}-n_{c l, e f f}\right) \Lambda
$$

where $\lambda_{\text {res }}$ is the resonance wavelength, $\Lambda$ is the grating pitch, and $n_{c o, \text { eff }}$ and $n_{c l \text { eff }}$ are the effective refractive indices of the fundamental core and cladding modes of the LPFG, respectively. ${ }^{(18)}$ Owing to the birefringence of the HBPCF between its two principal axes (fast and slow axes), caused by its asymmetric cladding structure and large index difference between air and glass, $\lambda_{\text {res }}$ satisfying (1) depends on the input PS. This is because the ratio of optical power coupled to the fast and slow axes of the HBPCF is different; thus, $n_{c o, e f f}$ and $n_{c l, e f f}$ have different values according to input polarization. As a result, the transmission spectra of the THBPC-LPFG have two rejection bands with different $\lambda_{\text {res }}$ 's with respect to orthogonal input PSs aligned along the slow and fast axes of the HBPCF. The band rejection ratio (BRR) of the 
THBPC-LPFG directly depends on the input PS, because its BRR is determined by the coupling ratio of input light into its two principal axes. ${ }^{(16)}$ If one of the two principal axes is excited, one of the two rejection bands with the maximum BRRs at their resonance wavelengths is chosen. In particular, these two rejection bands bound within several tens of nanometers, obtained at orthogonal input PSs, can have different cladding-mode orders. ${ }^{(16)}$

Figure 2 shows the input-polarization-dependent transmission spectra of the fabricated THBPC-LPFG, measured for two orthogonal input PSs denoted by polarizer angles of 0 and $90^{\circ}$, displayed as violet and orange solid lines, respectively, at room temperature without any applied strain. The input PS was set by a polarization controller (PC, Agilent 8169) composed of a linear polarizer, a quarter-wave plate (QWP), and a half-wave plate (HWP). The orientation angles of the polarizer, the QWP, and the HWP of the PC were controlled to excite two principal axes of the THBPC-LPFG. The polarizer angles of 0 and $90^{\circ}$ in Fig. 2 stand for these angular arrangements of the PC to excite the fast and slow axes (or vice versa) of the THBPC-LPFG, respectively. As can be seen from the figure, not one resonance dip but multiple attenuation dips are observed in each rejection band. This spectral splitting of the resonance dip can be understood as follows. When the fiber is under tension, a relatively weak $\mathrm{CO}_{2}$ laser radiation is sufficient to induce a significant index change in the cladding through the effect of frozenin viscoelasticity. ${ }^{(19)}$ Frozen-in viscoelasticity is the effect of freezing inelastic strains in a fiber during the fiber draw or by the heat treatment of a post-draw fiber under tension. ${ }^{(20)}$ In our study, the HBPCF is under twisting tension during the $\mathrm{CO}_{2}$ laser irradiation. Hence, $\mathrm{CO}_{2}$ laser heating produces a frozen-in strain in the twisted HBPCF at the point of irradiation. This locally induced strain turns into a torsion stress, when untwisting the HBPCF after the grating inscription. This torsion stress frozen in the HBPCF makes each rejection band spectrally split. No wavelength splitting was observed in an LPFG fabricated by simply rotating the fiber exposed to $\mathrm{CO}_{2}$ laser radiation; ${ }^{(21)}$ frozen-in strains do not exist in such an LPFG. Dips 1 and 2,

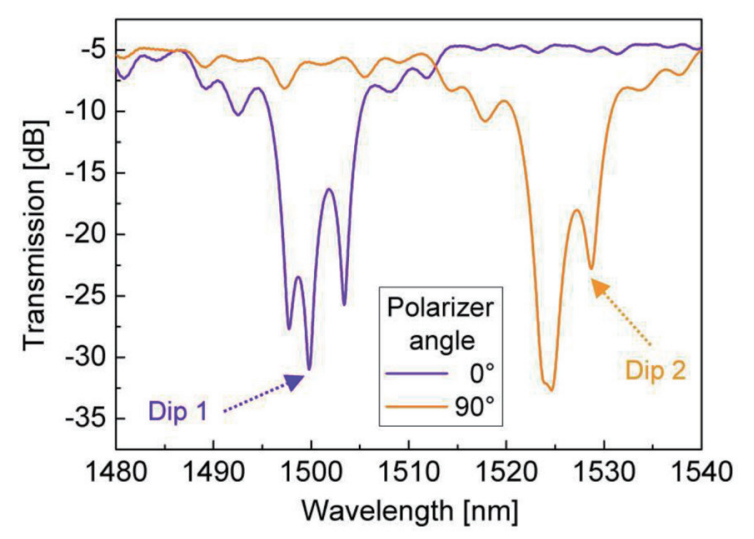

Fig. 2. (Color online) Input-polarization-dependent transmission spectra of the fabricated THBPC-LPFG, measured for two orthogonal input PSs denoted by polarizer angles of 0 and $90^{\circ}$, displayed as violet and orange solid lines, respectively. Dips 1 and 2 indicate two pointed dips selected as sensor indicators in the two attenuation bands of the THBPC-LPFG, obtained at polarizer angles of 0 and $90^{\circ}$, respectively. 
displayed as violet and orange solid lines, are sensor indicators selected at two rejection bands of the fabricated THBPC-LPFG, obtained at polarizer angles of 0 and $90^{\circ}$, respectively. The wavelengths $\lambda_{1}$ and $\lambda_{2}$ of Dips 1 and 2 were measured to be $\sim 1499.8$ and $\sim 1528.7 \mathrm{~nm}$ at polarizer angles of 0 and $90^{\circ}$, respectively. Moreover, the BRRs of Dips 1 and 2 were measured to be larger than 26 and $18 \mathrm{~dB}$, respectively.

Most attenuation dips of an LPFG show a highly linear strain or temperature response, regardless of the shift direction (i.e., red or blue shift). Resonance dips with different claddingmode orders have different strain or temperature sensitivities, ${ }^{(22)}$ and two rejection bands of the THBPC-LPFG, obtained at two orthogonal input PSs, can have different cladding-mode orders. If Dips 1 and 2 have different strain or temperature sensitivities, which implies that their responses are independent, strain and temperature changes (i.e., $\Delta S$ and $\Delta T$, respectively) simultaneously applied to the THBPC-LPFG can be discriminated and calculated using the following relation:

$$
\left[\begin{array}{c}
\Delta \lambda_{1} \\
\Delta \lambda_{2}
\end{array}\right]=\left[\begin{array}{ll}
K_{S, 1} & K_{T, 1} \\
K_{S, 2} & K_{T, 2}
\end{array}\right]\left[\begin{array}{c}
\Delta S \\
\Delta T
\end{array}\right] .
$$

Here, $\Delta \lambda_{1}$ and $\Delta \lambda_{2}$ indicate the perturbation-induced wavelength shifts of Dips 1 and 2 , respectively. The sensitivity coefficients $K_{S, 1}$ and $K_{S, 2}$ imply the strain sensitivities (i.e., $\Delta \lambda_{1} / \Delta S$ and $\Delta \lambda_{2} / \Delta S$ ) of Dips 1 and 2, respectively, when $\Delta T=0$. Similarly, $K_{T, 1}$ and $K_{T, 2}$ denote the temperature sensitivities (i.e., $\Delta \lambda_{1} / \Delta T$ and $\Delta \lambda_{2} / \Delta T$ ) of Dips 1 and 2, respectively, when $\Delta S=0$. These four sensitivity coefficients can be predetermined from the independent strain or temperature response measured for each sensor indicator dip (i.e., Dip 1 or 2). Hence, if strain- and temperature-induced $\Delta \lambda_{1}$ and $\Delta \lambda_{2}$ are measured from the transmission spectra of the THBPC-LPFG, $\Delta S$ and $\Delta T$ can be found through the following relation (3) using these predetermined sensitivity coefficients. That is, simultaneous measurements of strain and temperature can be achieved by measuring only $\Delta \lambda_{1}$ and $\Delta \lambda_{2}$.

$$
\left[\begin{array}{c}
\Delta S \\
\Delta T
\end{array}\right]=\frac{1}{|D|}\left[\begin{array}{cc}
K_{T, 2} & -K_{T, 1} \\
-K_{S, 2} & K_{S, 1}
\end{array}\right]\left[\begin{array}{l}
\Delta \lambda_{1} \\
\Delta \lambda_{2}
\end{array}\right]
$$

where $D=K_{S, 1} K_{T, 2}-K_{T, 1} K_{S, 2}$ is the determinant of the sensitivity coefficient matrix.

\section{Experimental Results and Discussion}

To investigate the independent strain or temperature response of the fabricated THBPCLPFG, an experimental setup was constructed using a BBS, a PC, an OSA with a resolution bandwidth of $0.02 \mathrm{~nm}$, a metal block, a translation stage, and a hot plate, as shown in Fig. 1. First, we utilized an adhesive to attach one end of the sensor head (i.e., the THBPC-LPFG) to a fixed metal block and the other end to the translation stage with an axial resolution of $5 \mu \mathrm{m}$. The longitudinal strain applied to the THBPC-LPFG could be adjusted by moving the translation 
stage. The distance $L_{0}$ between the metal block and the translation stage was set at $\sim 92 \mathrm{~mm}$. If the axial displacement of the translation stage is given by $\Delta L$, the longitudinal strain applied to the THBPC-LPFG can be expressed as $\Delta L / L_{0}$. Figures 3(a) and 3(b) show the strain-induced spectral variations of Dips 1 and 2 of the fabricated THBPC-LPFG (obtained at polarizer angles of 0 and $\left.90^{\circ}\right)$, measured for an applied strain from 0 to $1953 \mu \varepsilon$ at room temperature $\left(25^{\circ} \mathrm{C}, \Delta T=0\right)$, respectively. As can be seen from the figure, Dips 1 and 2 exhibit blue shifts (in the direction of blue arrows) of $\sim 1.92$ and $\sim 2.48 \mathrm{~nm}$ with increasing applied strain, respectively. Figures 3(c) and 3(d) show the resonance wavelength shifts of Dips 1 and 2, indicated by violet diamonds and orange circles, respectively. Solid lines in Figs. 3(c) and 3(d) indicate the linear fits of measured data points. It can be observed from the figures that Dips 1 and 2 show sufficiently linear strain responses with adjusted $R^{2}$ values of $\sim 0.99845$ and $\sim 0.99874$, respectively. The strain sensitivities of Dips 1 and 2, i.e., $K_{S, 1}$ and $K_{S, 2}$, were calculated from the measurement results to be approximately -0.99 and $-1.29 \mathrm{pm} / \mu \varepsilon$, respectively, which are comparable to those

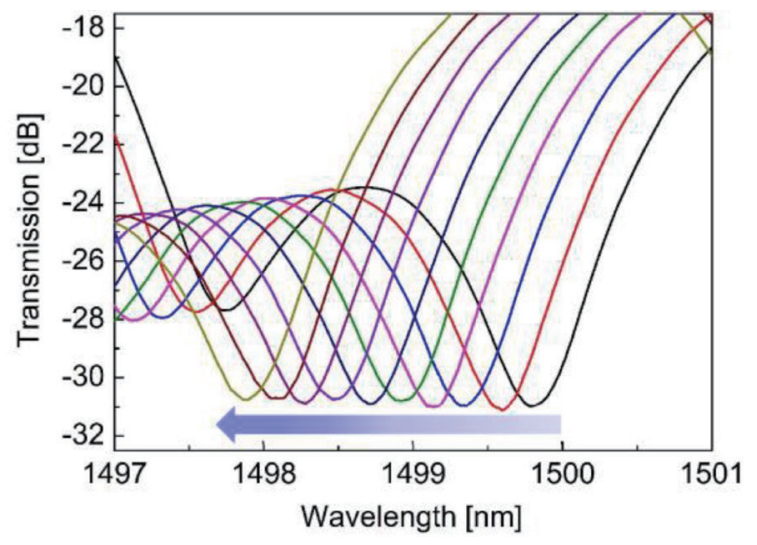

(a)

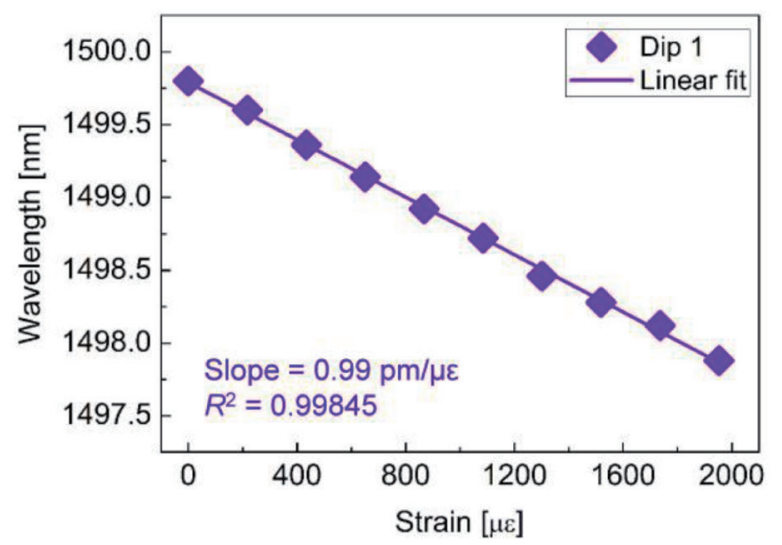

(c)

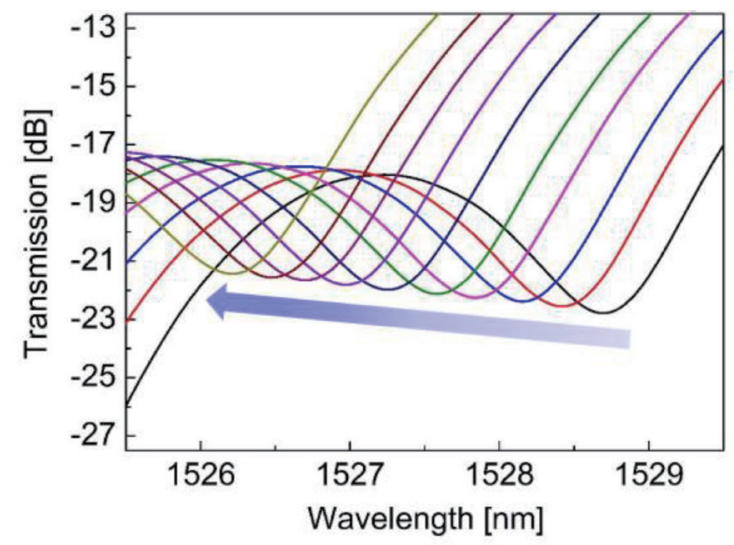

(b)

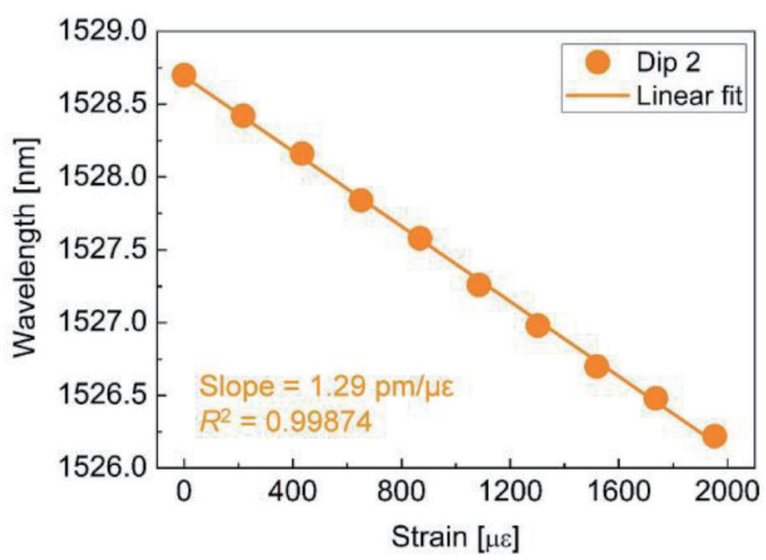

(d)

Fig. 3. (Color online) Strain-induced spectral variations of Dips (a) 1 and (b) 2 of the fabricated THBPCLPFG, measured for an applied strain from 0 to $1953 \mu \varepsilon$ at room temperature $\left(25^{\circ} \mathrm{C}, \Delta T=0\right)$, and the resonance wavelength shifts of Dips (c) 1 and (d) 2, indicated by violet diamonds and orange circles, respectively. Solid lines in (c) and (d) indicate the linear fits of measured data points. 
of the HB-LPFG. ${ }^{(16)}$ Here, $K_{S, 2}$ is $\sim 1.3$ times greater than $K_{S, 1}$, which implies that both rejection bands containing Dips 1 and 2 have different cladding-mode orders.

To examine the thermal responses of Dips 1 and 2 of the THBPC-LPFG, the fabricated THBPC-LPFG was placed on a hot plate, as shown in Fig. 1, and the spectral variations of Dips 1 and 2 were monitored in a temperature range of 35 to $95{ }^{\circ} \mathrm{C}$ (step: $10^{\circ} \mathrm{C}$ ) without the applied strain $(\Delta S=0)$. During the temperature response investigation, a weight of $15 \mathrm{~g}$ was suspended on one side of the HBPCF segment containing the THBPC-LPFG with its other side fixed, to keep the sensor head straight. Figures 4(a) and 4(b) show the temperature-induced spectral variations of Dips 1 and 2 of the fabricated THBPC-LPFG (obtained at polarizer angles of 0 and $\left.90^{\circ}\right)$, measured for an applied temperature from 35 to $95{ }^{\circ} \mathrm{C}$ without the applied strain $(\Delta S=0)$, respectively. As can be observed from the figure, Dips 1 and 2 show red shifts (in the direction of red arrows) of $\sim 0.42$ and $\sim 0.60 \mathrm{~nm}$ with increasing ambient temperature, respectively. Figures 4(c) and 4(d) show the resonance wavelength shifts of Dips 1 and 2, indicated by violet diamonds and orange circles, respectively.

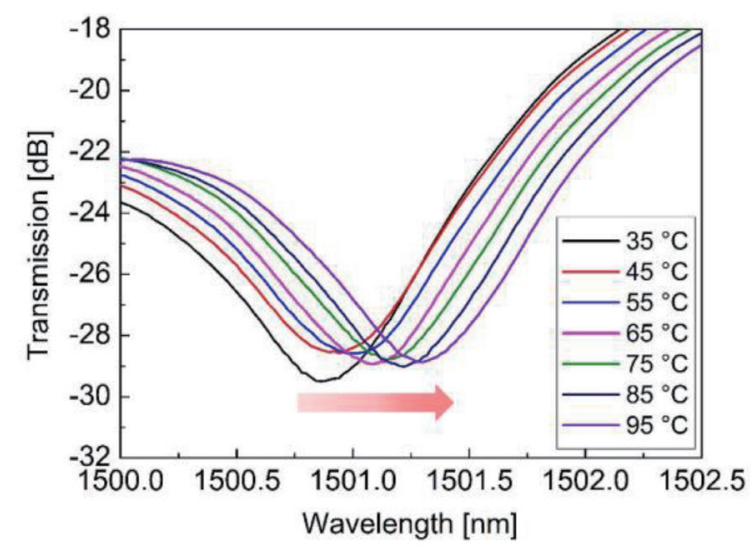

(a)

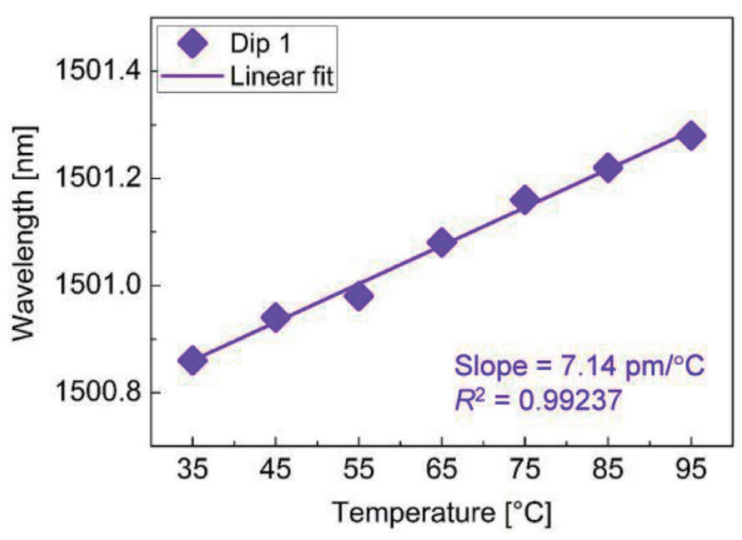

(c)

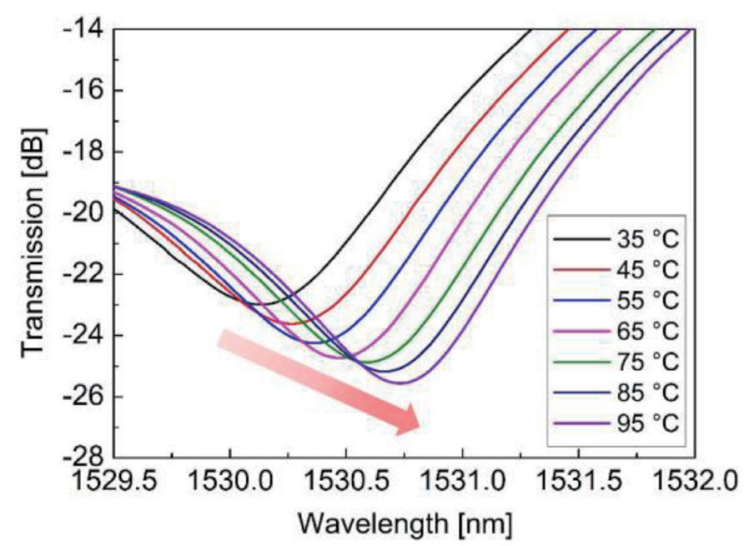

(b)

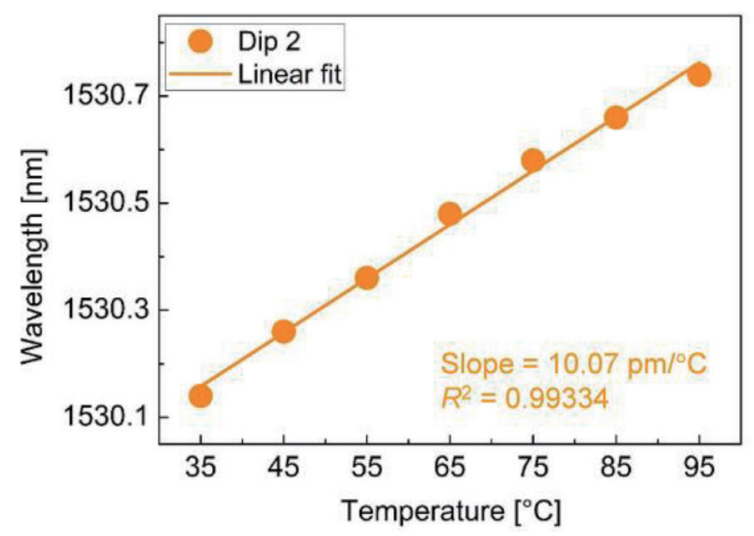

(d)

Fig. 4. (Color online) Temperature-induced spectral variations of Dips (a) 1 and (b) 2 of the fabricated THBPCLPFG, measured for an applied temperature from 35 to $95{ }^{\circ} \mathrm{C}$ without applied strain $(\Delta S=0)$, and the resonance wavelength shifts of Dips (c) 1 and (d) 2, indicated by violet diamonds and orange circles, respectively. Solid lines in (c) and (d) indicate the linear fits of measured data points. 
Similarly, solid lines in Figs. 4(c) and 4(d) indicate the linear fits of measured data points. It can be found from the figures that Dips 1 and 2 also show sufficiently linear temperature responses with adjusted $R^{2}$ values of $\sim 0.99237$ and $\sim 0.99334$, respectively. The temperature sensitivities of Dips 1 and 2, i.e., $K_{T, 1}$ and $K_{T, 2}$, were evaluated to be $\sim 7.14$ and $\sim 10.07$ $\mathrm{pm} /{ }^{\circ} \mathrm{C}$, respectively. Here, $K_{T, 2}$ is $\sim 1.4$ times higher than $K_{T, 1}$, which also corroborates that both rejection bands containing Dips 1 and 2 have different cladding-mode orders. These temperature sensitivities of the THBPC-LPFG are much smaller than those of the HB-LPFG. ${ }^{(16)}$ This is because the HBPCF consists of pure silica, a single substance. However, the lower temperature sensitivity does not make the simultaneous measurements of strain and temperature impossible. On the basis of the measurement results of the four sensitivity coefficients $\left(K_{S, 1}\right.$, $K_{S, 2}, K_{T, 1}$, and $K_{T, 2}$ ), Eq. (3) can be rewritten as

$$
\left[\begin{array}{l}
\Delta S \\
\Delta T
\end{array}\right]=\frac{1}{0.7587}\left[\begin{array}{cc}
10.07 & -7.14 \\
1.29 & -0.99
\end{array}\right]\left[\begin{array}{l}
\Delta \lambda_{1} \\
\Delta \lambda_{2}
\end{array}\right]
$$

The different magnitudes and signs of these four sensitivity coefficients are attributed to the core and cladding effective refractive indices, the waveguide structure, and strain- or thermooptic coefficients of the HBPCF, which are also polarization-dependent. By measuring the wavelength shifts $\left(\Delta \lambda_{1}\right.$ and $\left.\Delta \lambda_{2}\right)$ of Dips 1 and 2, one can use Eq. (4) to directly find the applied strain and temperature.

Finally, we carried out simultaneous measurements of strain and temperature by ten times for specific variations of the applied strain and temperature by using the THBPC-LPFG. The simultaneous measurements were performed for three different applied strain variations $(\Delta S$ $=651,868$, and $1085 \mu \varepsilon)$ and three different applied temperature variations $(\Delta T=0,20$, and $\left.40{ }^{\circ} \mathrm{C}\right)$. Figure 5 shows the simultaneous measurement results for nine $(\Delta S, \Delta T)$ points, which were obtained by analyzing the measured transmission spectra. The error bars in the measured points indicate the maximum deviations from the applied parameter values. The rms deviations of the measured strain and temperature were $20.2 \mu \varepsilon$ and $1.78{ }^{\circ} \mathrm{C}$, respectively. It is found

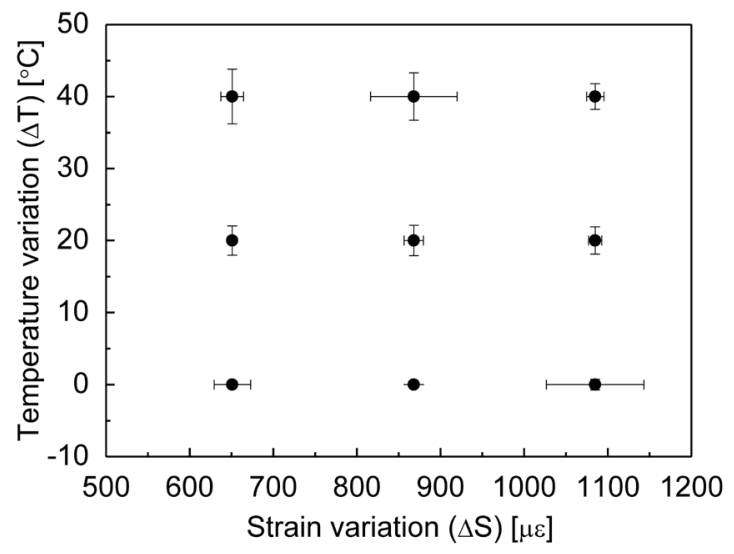

Fig. 5. Simultaneous measurement results for nine $(\Delta S, \Delta T)$ points, which were obtained for three different applied strain variations $(\Delta S=651,868$, and $1085 \mu \varepsilon)$ and three different applied temperature variations $(\Delta T=0,20$, and $40{ }^{\circ} \mathrm{C}$ ). 
from these measurement results that the proposed sensor can perform separate measurements of strain and temperature with sufficient measurement accuracy. In brief, the THBPC-LPFG can be employed as a bend-insensitive sensor head capable of simultaneously discriminating between strain and temperature.

\section{Conclusion}

We demonstrated a fiber-optic sensor capable of separately measuring strain and temperature by using a THBPC-LPFG as a sensor head. The THBPC-LPFG was manufactured by irradiating $\mathrm{CO}_{2}$ laser pulses on a twisted HBPCF. The fabricated THBPC-LPFG had two different rejection bands, within which multiple attenuation dips existed, with respect to two orthogonal input PSs. These two rejection bands were closely spaced and had different cladding-mode orders. Two attenuation dips (i.e., Dips 1 and 2), each of which was selected in each rejection band, were utilized as sensor indicators. The strain and temperature responses of these two indicators were investigated in a strain range of 0 to $1953 \mu \varepsilon$ and in a temperature range of 35 to $95{ }^{\circ} \mathrm{C}$. The two dips showed linear and independent responses to the applied strain and temperature, and their strain and temperature sensitivities were evaluated from the measurement results as approximately $-0.99 \mathrm{pm} / \mu \varepsilon$ and $7.14 \mathrm{pm} /{ }^{\circ} \mathrm{C}$ for Dip 1 and $-1.29 \mathrm{pm} / \mu \varepsilon$ and $10.07 \mathrm{pm} /{ }^{\circ} \mathrm{C}$ for Dip 2, respectively. Using these four sensitivity coefficients, strain and temperature changes applied to the THBPC-LPFG can be simultaneously drawn out from the measured wavelength shifts of the two sensor indicators (Dips 1 and 2). From the repetitive simultaneous measurements for nine $(\Delta S, \Delta T)$ points, the rms deviations of the measured strain and temperature were evaluated as $20.2 \mu \varepsilon$ and $1.78{ }^{\circ} \mathrm{C}$, respectively. This implies that the fabricated THBPC-LPFG can be used as a bend-insensitive sensor head for the simultaneous measurements of strain and temperature.

\section{Acknowledgments}

This research was supported by the Basic Science Research Program through the National Research Foundation of Korea (NRF) funded by the Ministry of Education (2019R1I1A3A01046232).

\section{References}

1 Y. Liu, J. A. R. Williams, and I. Bennion: IEEE Photonics Technol. Lett. 12 (2000) 531. http://dx.doi. org/10.1109/68.841276

2 Y. P. Wang and Y. J. Rao: IEEE Sens. J. 5 (2005) 839. http://dx.doi.org/10.1109/JSEN.2005.844335

3 M. Deng, C. P. Tang, T. Zhu, and Y. J. Rao: Opt. Commun. 284 (2011) 2849. https://doi.org/10.1016/ j.optcom.2011.02.061

4 H. J. Patrick, A. D. Kersey, and F. Bucholtz: J. Lightwave Technol. 16 (1998) 1606. https://www.osapublishing. org/jlt/abstract.cfm?URI=jlt-16-9-1606

5 F. Zou, Y. Liu, C. Deng, Y. Dong, S. Zhu, and T. Wang: Opt. Express 23 (2015) 1114. https://doi.org/10.1364/ OE.23.001114

6 M. Konstantaki, S. Pissadakis, S. Pispas, N. Madamopoulos, and N. A. Vainos: Appl. Opt. 45 (2006) 4567. https://doi.org/10.1364/AO.45.004567

7 A. Urrutia, J. Goicoechea, A. L. Ricchiuti, D. Barrera, S. Sales, and F. J. Arregui: Sens. Actuator, B 227 (2016) 135. https://doi.org/10.1016/j.snb.2015.12.031 
8 C. Y. Lin, L. A. Wang, and G. W. Chern: J. Lightwave Technol. 19 (2001) 1159. https://www.osapublishing.org/ jlt/abstract.cfm?URI=jlt-19-8-1159

9 Y. P. Wang, L. Xiao, D. N. Wang, and W. Jin: Opt. Lett. 31 (2006) 3414. https://doi.org/10.1364/OL.31.003414

10 T. Guan, Z. Gu, Q. Ling, and W. Feng: Opt. Laser Technol. 114 (2019) 20. https://doi.org/10.1016/ j.optlastec.2019.01.026

11 X. Shu, T. Allsop, B. Gwandu, L. Zhang, and I. Bennion: IEEE Photonics Technol. Lett. 13 (2001) 818. https:// doi.org/10.1109/68.935814

12 S. Khaliq, S. W. James, and R. P. Tatam: Meas. Sci. Technol. 13 (2002) 792. https://stacks.iop.org/MST/13/792

13 D. Yang, P. Zhang, J. Zeng, X. Wang, B. Song, and S. Dai: J. Lightwave Technol. 35 (2017) 3974. https://doi. org/10.1109/JLT.2017.2725302

14 V. Bhatia, D. Campbell, R. O. Claus, and A. M. Vengsarkar: Opt. Lett. 22 (1997) 648. https://doi.org/10.1364/ OL.22.000648

15 Y. G. Han, S. B. Lee, C. S. Kim, J. U. Kang, U. C. Paek, and Y. Chung: Opt. Express 11 (2003) 476. https://doi. org/10.1364/OE.11.000476

16 K. J. Han, Y. W. Lee, J. Kwon, S. Roh, J. Jung, and B. Lee: IEEE Photonics Technol. Lett. 16 (2004) 2114. https://doi.org/10.1109/LPT.2004.833081

17 T. Zhu, K. S. Chiang, Y. J. Rao, C. H. Shi, Y. Song, and M. Liu: J. Lightwave Technol. 27 (2009) 4863. https:// www.osapublishing.org/jlt/abstract.cfm?URI=jlt-27-21-4863

18 A. M. Vengsarkar, P. J. Lemaire, J. B. Judkins, V. Bhatia, T. Erdogan, and J. E. Sipe: J. Lightwave Technol. 14 (1996) 58. https://doi.org/10.1109/50.476137

19 Y. Liu and K. S. Chiang: Opt. Lett. 33 (2008) 1933. https://doi.org/10.1364/OL.33.001933

20 A. D. Yablon: IEEE J. Sel. Top. Quantum Electron. 10 (2004) 300. http://doi.org/10.1109/JSTQE.2004.826570

21 S. Oh, K. R. Lee, U. C. Paek, and Y. Chung: Opt. Lett. 29 (2004) 1464. https://doi.org/10.1364/OL.29.001464

22 X. Shu, L. Zhang, and I. Bennion: J. Lightwave Technol. 20 (2002) 255. https://doi.org/10.1109/50.983240

\section{About the Authors}

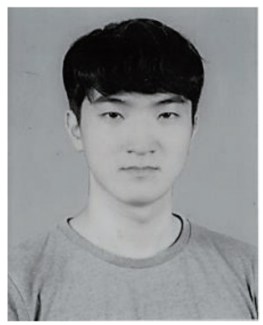

Do Kyung Kim received his B.S. degree from the School of Electrical Engineering, Pukyong National University, Busan, South Korea in 2017, where he is currently pursuing his master's degree under the Interdisciplinary Program of Biomedical Mechanical and Electrical Engineering. His research interests include optical fiber devices for optical sensors and filters.

(dosokre@naver.com)

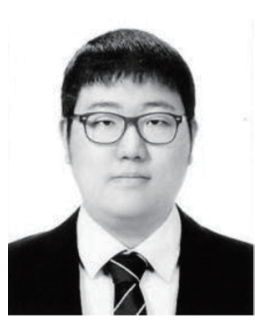

Jihoon Kim received his B.S. and M.S. degrees from the School of Electrical Engineering, Pukyong National University, Busan, South Korea, in 2013 and 2015, respectively, where he is currently pursuing his Ph.D. degree. His current research interests include current switching in semiconductor thin films based on photo-induced phase transition and optical devices for optical sensors and communications, such as optical fiber gratings and filters. (ghkim613@gmail.com)

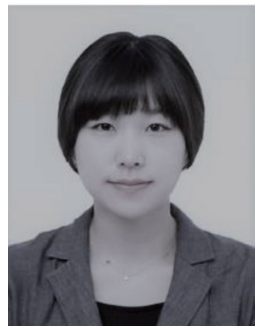

Seul-Lee Lee received her B.S. and M.S. degrees from the School of Electrical Engineering, Pukyong National University, Busan, South Korea, in 2012 and 2014, respectively, where she is currently pursuing her Ph.D. degree under the Interdisciplinary Program of Biomedical Mechanical and Electrical Engineering. Her research interests include optical fiber devices for optical sensors and communications, such as optical fiber gratings and optical fiber lasers. (kls1116@hanmail.net) 


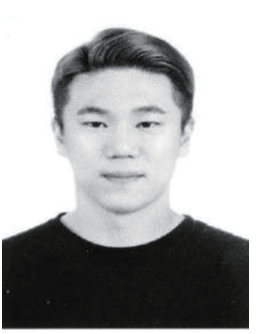

Sungwook Choi received his B.S. and M.S. degrees from the School of Electrical Engineering and the Interdisciplinary Program of Biomedical Mechanical and Electrical Engineering, Pukyong National University, Busan, South Korea, in 2015 and 2017, respectively, where he is currently pursuing his Ph.D. degree under the Interdisciplinary Program of Biomedical Mechanical and Electrical Engineering. His research interests include optical fiber sensors and switching devices based on oxide semiconductor thin films. (leewan777@gmail.com)

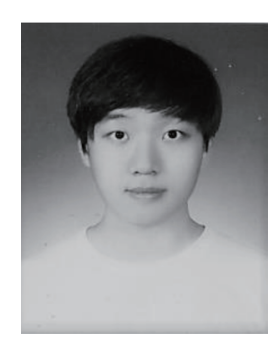

Min Seok Kim received his B.S. degree from the School of Electrical Engineering, Pukyong National University, Busan, South Korea in 2017, where he is currently pursuing his master's degree under the Interdisciplinary Program of Biomedical Mechanical and Electrical Engineering. His research interests include optical fiber lasers, optical fiber sensors, and electronic sensors based on thin-film semiconductors. (doragee91@naver.com)

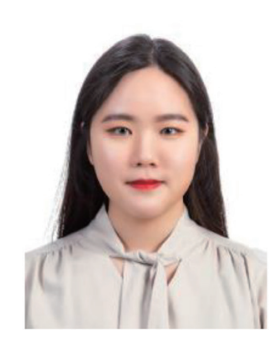

Jinsil Han received her B.S. degree from the School of Electrical Engineering, Pukyong National University, Busan, South Korea, in 2020, where she is currently pursuing her master's degree under the Interdisciplinary Program of Biomedical Mechanical and Electrical Engineering. Her research interests include fiber-optic physical, chemical, and biosensors.

(jinsi13208@pukyong.ac.kr)

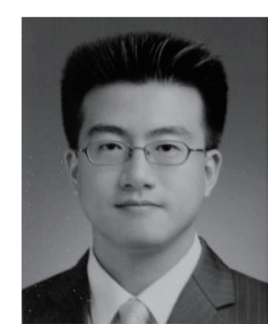

Yong Wook Lee received his B.S., M.S., and Ph.D. degrees from the School of Electrical Engineering, Seoul National University, Seoul, South Korea, in 1998, 2000, and 2004, respectively. From 2004 to 2008, he was a senior researcher at the Electronics and Telecommunications Research Institute, Daejeon, South Korea. He is currently a professor at the School of Electrical Engineering, Pukyong National University, Busan, South Korea. He is the author of more than 100 journal papers. His research interests include optical devices for optical sensors and communications, such as optical fiber gratings, optical filters, optical amplifiers, and optical switching in semiconductor thin films based on photo-induced phase transition. (yongwook@pknu.ac.kr) 